\title{
Self-diagnosing braided composite rod
}

\author{
E. Zdraveva \& R. Fangueiro \\ Department of Textile Engineering, University of Minho, Portugal \\ C. Gonilho-Pereira \\ Department of Civil Engineering, University of Minho, Portugal
}

A. Ferreira \& S. Lanceros-Mendez

Department of Physics, University of Minho, Portugal

\begin{abstract}
This paper presents the development of a braided reinforced composite rod (BCR) able to both reinforce and monitor the stress state of concrete structures. Carbon fibers have been used as sensing and reinforcing materials along with glass fiber. Various composites rods have been produced using an author patented technique based on a modified conventional braiding machine. The materials investigated were prepared with different carbon fiber content as follows: $\mathrm{BCR}_{2}\left(77 \%\right.$ glass $/ 23 \%$ carbon fiber), $\mathrm{BCR}_{3}(53 \%$ glass $/ 47 \%$ carbon fiber $), \mathrm{BCR}_{4}$ (100\% carbon fiber). BCRs have been tested under bending while the variation of the electrical resistance was simultaneously monitored. The correlations obtained between deformation and electrical resistance show the suitability of the rods to be used as sensors. The fractional resistance change versus strain plots show that the gage factor increases with decreasing carbon fiber content.
\end{abstract}

\section{INTRODUCTION}

External dynamic loading and severe environmental conditions are the major cause of deterioration in reinforced concrete structures. This deterioration may cause serious structural damages. The corrosion of steel reinforcing rebar is the dominant cause of concrete structure degradation. The most effective way to prevent corrosion of steel rebar is the use of a corrosion resistant reinforcing material, such as fiber-reinforced-polymer (FRP) composites. The types of fiber-reinforced-polymer composites best suited for the reinforcement of concrete are those providing high strength, high stiffness, and environmental compatibility with concrete. Most commercial FRPs are rod-like elements that are pultruded, shaped, and treated so that surface texture provide mechanical adherence with concrete.

Nevertheless, the interest in the safety of concrete structures has increased and monitoring and maintaining their safety has become a main goal. To achieve this main goal monitoring systems that can be applied to the reinforced concrete elements are required. The damage sensing is conventionally performed by attached or embedded damage sensors, such as optical fibers, acoustic sensors, etc. however these sensors have limited application because of high cost, low durability, and limited sensing volume and spatial resolution. One solution is that the materials themselves can possess a self-diagnosing function for fracture; thus, strong and heavy design, complex and expensive equipment and numerous sensors becomes unnecessary, the so called self-diagnosing structural materials (Muto, 2001), (Chung, 1997).

Structural materials have evolved from materials that are mechanically strong (such as steel) to materials that are both strong and lightweight (such as composite materials) and most recently to materials that are both strong and self-monitoring (Muto, 2001). By definition, a selfmonitoring material is one which can sense its own strain and damage. It can be considered a smart material. However, in contrast to smart materials such as optical fibers, piezoelectric sensors, etc., the self-monitoring materials are themselves structural materials. Thus, in contrast to structures rendered smart by embedded or attached sensors, self-diagnosing structural materials 
are intrinsically smart, so there is no need of embedded or attached sensors. For example, the basic principle of the carbonaceous smart structural material to detect strain or damage lies in the electrical conductivity of the carbon fibers, as already known from the literature (Chung, 1997). As the carbon fibres are electrically conductive, the composite itself can exhibit electrical properties, which will depend upon strain, damage and temperature. The self-diagnosing structural material will, in this way, provide determination of the strain or damage by measuring the change in the electrical resistance during real time loading (Bakis, 2001).

Several investigations carried on this subject are as follow: hybrid, carbon fibre (CF) and glass fibre (GF) reinforced composite rods were tested for sensing performance. It was reported that the memorizing ability of the material resulted from the increased residual resistance of the composites after loading-unloading cycles, which was in dependence with the previously applied maximum strain. Under pre-stressed condition the composite gave remarkable memorizing ability with lower detectable strains. A main drawback raised by this study is related to damage detection: damage cannot be detected in the early stages, unless the carbon fibres are replaced with carbon particles (Okuhara, 2005). In the later case, on the other hand, massive production is more expensive and demanding.

Another study using a glass fibre reinforced composite rod incorporating carbon particles dispersed in the resin reported that the material can be used as an autonomous sensor, as it can monitor strain without external pre-stress condition (Okuhara, 2007).

In a hybrid composite, the carbon fibre type and the relative percentage of carbon fiber and glass fiber are factors that determine the variations of the electrical resistance upon stress and/or strain. As mentioned before, the main drawback of hybrid CF-GF reinforced polymer composites is the lack of sensitivity at lower strains, which can be improved by pre-stressing (Nanni, 2007).

Nevertheless, by modifying fibre reinforced polymer composites (FRP) concentration formulations and geometry (e.g., carbon fibres core and glass fibres insulating outer layer components), the electrical response of the composites can be tailored to a desired value (Nanni, 2006)

In this work the monitoring capabilities of braided reinforced composite rods (BCRs) and the differences in the sensing behavior related to the different carbon fiber content are investigated.

\section{MATERIALS AND METHODS}

Three types of braided composite rods with different carbon fiber content $(23 \%, 77 \%$ and $100 \%$ ) were prepared by the single step process of braiding and simultaneous resin impregnation. Braided reinforced composite rods have been produced on a vertical braiding machine with an incorporated impregnation system (Gonilho Pereira, 2008). Table 1 shows the BCR composition while Figure 1 shows the correspondent BCR cross-sections. A total number of 14 samples were prepared and evaluated.

Table 1. BCR compositions.

\begin{tabular}{ccccc}
\hline Type & Fiber composition & Fiber \% & $\mathrm{N}^{\circ}$ of rovings & Linear mass (tex) \\
\hline $\mathrm{BCR}_{2}$ & E-glass/Carbon & $77 / 23$ & $18 / 3$ & $1600 / 900$ \\
$\mathrm{BCR}_{3}$ & E-glass/Carbon & $53 / 47$ & $53 / 47$ & 900 \\
$\mathrm{BCR}_{4}$ & Carbon & 100 & 12 & 900 \\
\hline
\end{tabular}
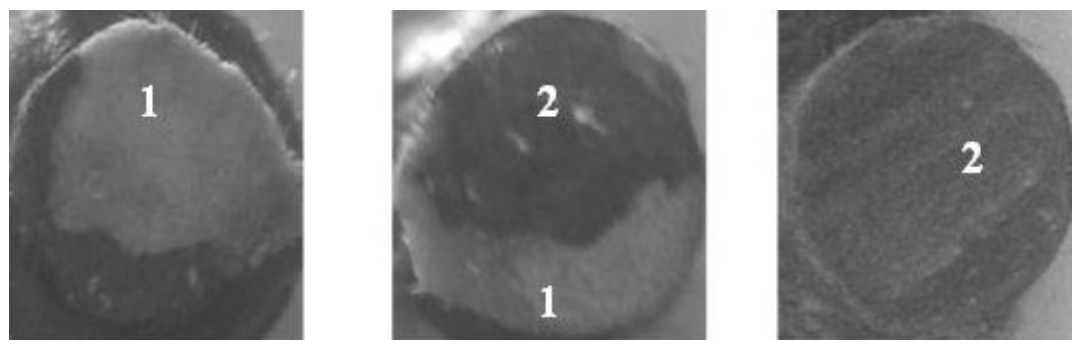

Figure 1. BCR cross-sections for $\mathrm{BCR}_{2}, \mathrm{BCR}_{3}$ and $\mathrm{BCR}_{4} .1$ - Glass fiber 2 -Carbon fiber. 
The testing procedure carried on the BCR is based on the electrical resistance measurement during simultaneous application of a deformation in a cyclic three-point bending test (Figure 2). The testing parameters are shown in Table 2. Cyclic three-point bending tests (Table 3) were carried on a Universal Testing Machine - Autograph IS (Shimadzu) 500N. The electrical resistance measurement was carried on a digital multi-meter (Agilent, 84401A). The electrical signal was acquired through golden wires attached to the cross section of the samples with silver paint.

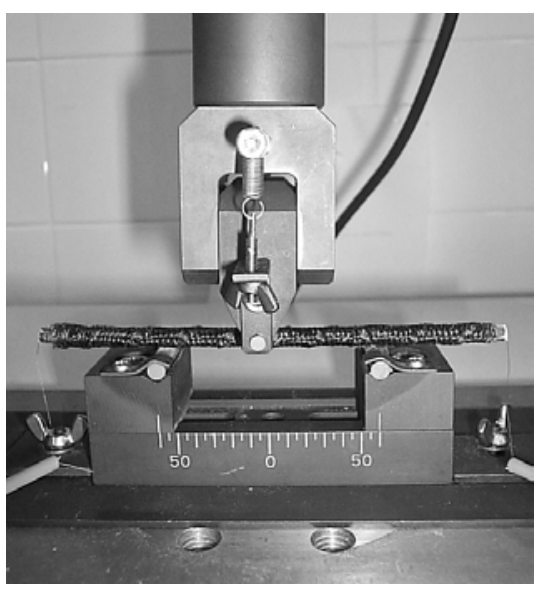

Figure 2. Sample set-up during testing.

Table 2. Dimensional characteristics of the BCR s.

\begin{tabular}{lcc}
\hline BCR type & Diameter $(\mathrm{mm})$ & Linear mass $(\mathrm{g} / \mathrm{m})$ \\
\hline $\mathrm{BCR}_{2}$ (glass 77\%, carbon 23\%) & 5,66 & 36,16 \\
$\mathrm{BCR}_{3}$ (glass 53\%, carbon 47\%) & 5,80 & 39,89 \\
$\mathrm{BCR}_{4}$ (carbon 100\%) & 6,40 & 40,47 \\
\hline
\end{tabular}

Table 3. Testing parameters.

\begin{tabular}{ll}
\hline Parameter & Settings \\
\hline $\mathrm{N}^{\circ}$ of cycles & 4 \\
Load cell & $500 \mathrm{~N}$ \\
Span & $6 \mathrm{~cm}$ \\
Displacement limit & $0,5 \mathrm{~mm}$ \\
Cross-head speed & $0,3 \mathrm{~mm} / \mathrm{min}$ \\
Sampling interval & $500 \mathrm{~ms}$ \\
\hline
\end{tabular}

\section{EXPERIMENTAL RESULTS AND DISCUSSION}

Representative examples of the two types of behavior obtained for the mechanical and electrical results from the cyclic loading three-point bending tests and the simultaneous electrical resistance measurements are presented in Figures 3 and 4 for the BCR samples tested.

Figure 3 presents the increase in the electrical resistance with increasing displacement. Figure 4 shows curves showing the decrease of the electrical resistance with increasing deformation. In general, the electrical resistance during loading and unloading increases linearly at lower displacement values and nonlinearly at higher ones. In the case of the inverse response, during decrease of the electrical resistance and deformation increase, the nonlinearity is less evident. Whether it was a reverse or inverse response in both cases the tested sample showed the change of the electrical resistance in proper compliance with the change of its deformation. The main factors influencing the type of response of each sample was the relative position of the fibers and the resin inside the rods, or more precisely the carbon fiber placement along the length of the rods. 


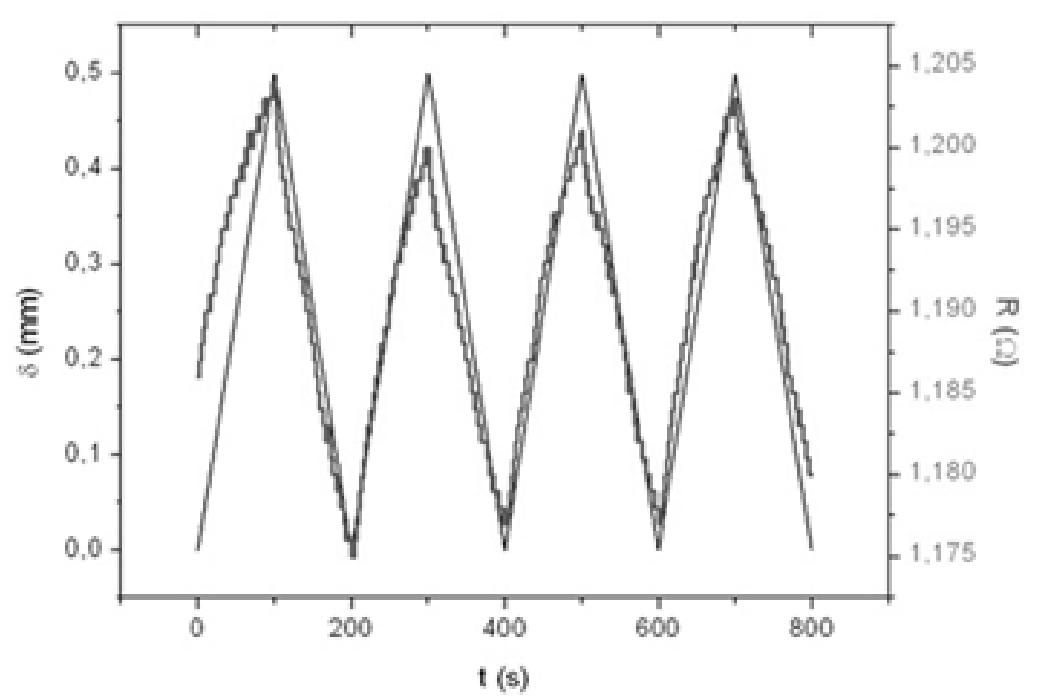

Figure 3. Displacement, resistance change and time dependence for type $\mathrm{BCR}_{4}(100 \%$ of carbon); Positive response of tested samples.

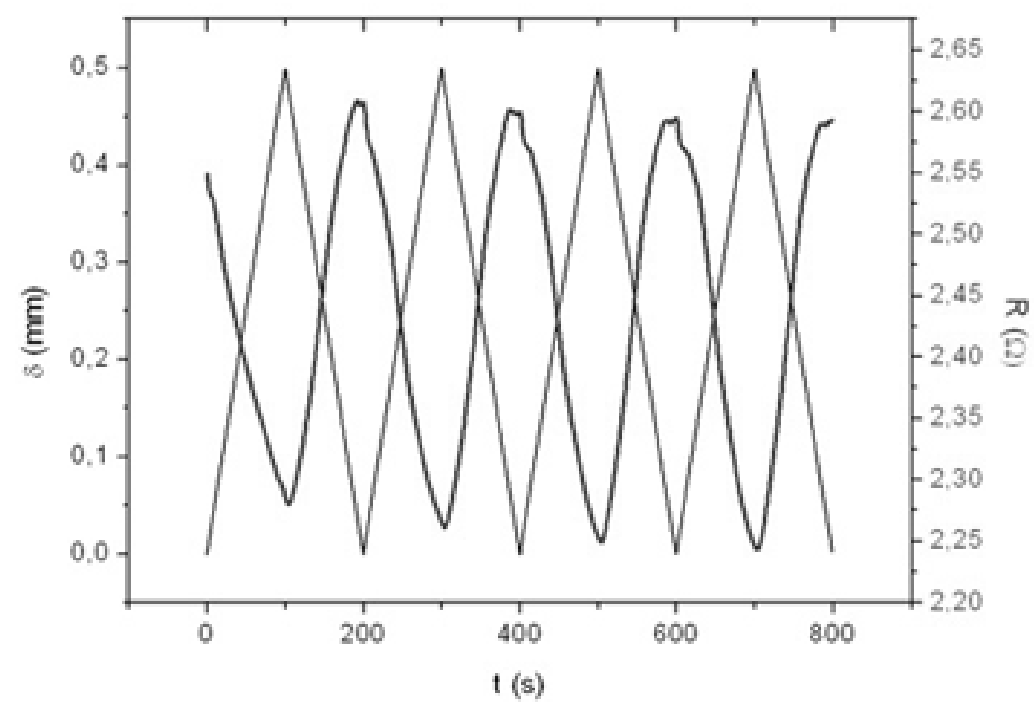

Figure 4. Displacement, resistance change and time dependence for type $\mathrm{BCR}_{2}(77 \%$ of glass, $23 \%$ of carbon); Negative response of tested samples.

As shown in Figure 1, from the images of the samples cross-sections, there are variations in the carbon fiber placement. The fibers are not placed uniformly on one side of the cross-section and, more important, the distribution is not the same on both cross-sections of a rod sample. In this situation, increasing of the resistance with increasing deformation occurred when the carbon fibers are placed on the tensile side and the opposite behavior when their placement is on the compression side of the bending rod. This issue indicates the relevance of controlling carbon placement uniformly along the length of the rod. This is a difficult step on the production process, which sometimes results in non-uniformity along the length of the rods as the braiding process rotates the fibers [8].

On the other hand, for each rod, the cycles are reproducible, confirming the reliable sensing property of the rods. The difference between the peak values in each of the cycles is around $0,01 \Omega$ to $0,02 \Omega$.

The range of the initial electrical resistance for the first typeBCR 2 ( $77 \%$ glass, $23 \%$ carbon), vary from $1,46 \Omega$ to $3,92 \Omega$, for the second type BCR $_{3}(53 \%$ glass, $47 \%$ carbon) from $1,11 \Omega$ to $1,50 \Omega$ and for the third $\mathrm{BCR}_{4}(100 \%$ carbon) from $0,80 \Omega$ to $1,37 \Omega$. 
Furthermore, for the comparison of the sensing behavior differentiation due to carbon fiber content, the strain $\varepsilon\left(\times 10^{-2}\right)$ and the fractional resistance change $\Delta R / R_{0}$ of the three types of $\mathrm{BCR}$ are presented in Table 3. The strain is calculated from the displacement and the fractional resistance change is calculated from the electrical resistance change, the values from the two parameters are presented over time of 100,300, 500 and 700 seconds.

Table 4. Strain and fractional resistance change of the BCR (mean values).

\begin{tabular}{|c|c|c|c|c|c|c|c|c|c|}
\hline \multirow[b]{2}{*}{ Type } & \multirow{2}{*}{$\begin{array}{c}\text { Cycle No. } \\
\text { t (s) }\end{array}$} & \multicolumn{2}{|c|}{$\begin{array}{c}1 \\
100\end{array}$} & \multicolumn{2}{|c|}{$\begin{array}{c}2 \\
300\end{array}$} & \multicolumn{2}{|c|}{$\begin{array}{c}3 \\
500\end{array}$} & \multicolumn{2}{|c|}{$\begin{array}{c}4 \\
700\end{array}$} \\
\hline & & $\begin{array}{c}\varepsilon \\
\times 10^{-2} \\
\end{array}$ & $\Delta \mathrm{R} / \mathrm{R}_{0}$ & $\begin{array}{c}\varepsilon \\
\times 10^{-2} \\
\end{array}$ & $\Delta \mathrm{R} / \mathrm{R}_{0}$ & $\begin{array}{c}\varepsilon \\
\times 10^{-2} \\
\end{array}$ & $\Delta \mathrm{R} / \mathrm{R}_{0}$ & $\begin{array}{c}\varepsilon \\
\times 10^{-2} \\
\end{array}$ & $\Delta \mathrm{R} / \mathrm{R}_{0}$ \\
\hline \multirow{3}{*}{$\mathrm{BCR}_{2}$} & $\mathrm{Xm}$ & & & & & & & & \\
\hline & $1,2,3$ & 0,48 & $-0,10$ & 0,48 & $-0,11$ & 0,48 & $-0,12$ & 0,48 & $-0,12$ \\
\hline & $12,13,14$ & 0,47 & 0,08 & 0,47 & 0,07 & 0,47 & 0,07 & 0,47 & 0,06 \\
\hline \multirow{2}{*}{$\mathrm{BCR}_{3}$} & $3,7,12$ & 0,48 & 0,04 & 0,48 & 0,02 & 0,48 & 0,01 & 0,48 & 0,01 \\
\hline & $6,10,13$ & 0,48 & $-0,06$ & 0,48 & $-0,07$ & 0,48 & $-0,07$ & 0,48 & $-0,07$ \\
\hline $\mathrm{BCR}_{4}$ & $5,7,12$ & 0,55 & 0,02 & 0,55 & 0,01 & 0,55 & 0,01 & 0,55 & 0,01 \\
\hline
\end{tabular}

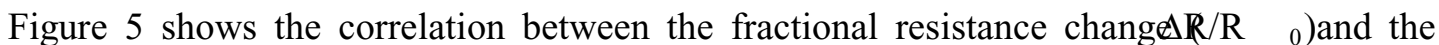
flexural deformation $(\varepsilon)$ for of all the three rod types $\mathrm{BCR}_{2}$ (77\% glass, $23 \%$ carbon), $\mathrm{BCR}_{3}$ (53\% glass, $47 \%$ carbon), $\mathrm{BCR}_{4}(100 \%$ carbon)in the time range $(0-100 \mathrm{sec} ; 200-300 \mathrm{sec} ; 400-$ $500 \mathrm{sec} ; 600-700 \mathrm{sec})$ - first half of the cycle (loading sequence), or (100-200sec; 300-400sec; $500-600 ; 700-800 \mathrm{sec}$ ) - second half of the cycle (unloading sequence).

Figures 5 A1 and 5 A2 represent the positive type of response. Figures 5 B1 and B2 represent the negative type of response. The numbers 1 and 2, respectively, represent the loading and unloading sequence of the cycles. Furthermore the trend lines are presented for each of the plotted curves showing the linear accordance between the fractional resistance change and the deformation.
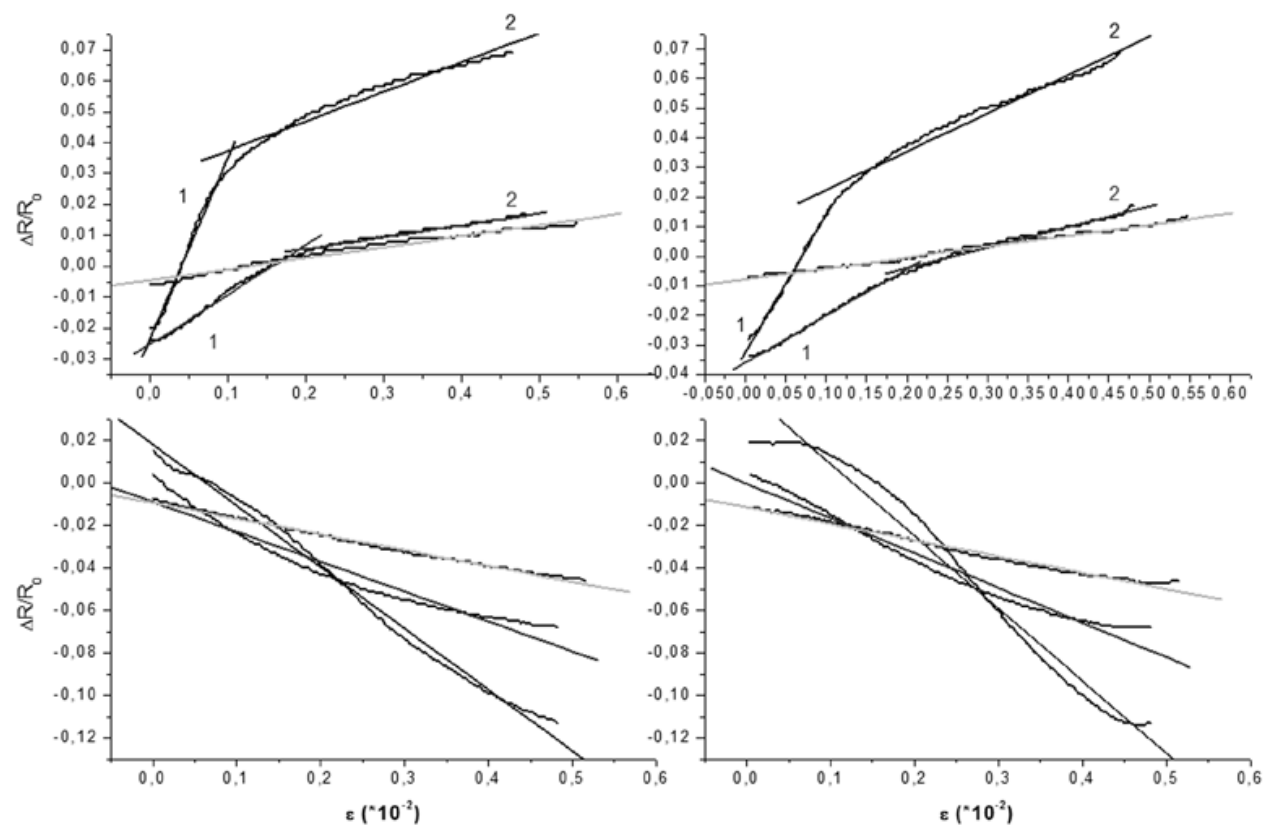

Figure 5. Fractional resistance change dependence on deformation. Comparison between the three types of tested samples: $\mathrm{BCR}_{2}, \mathrm{BCR}_{3}, \mathrm{BCR}_{4}$.
1 - loading
2 -unloading
A - positive response
$\mathrm{B}$ - negative response
$\mathrm{BCR}_{2}$ (77\% of glass, $23 \%$ of carbon) $\mathrm{BCR}_{3}(53 \%$ of glass, $47 \%$ of carbon) $\mathrm{BCR}_{4}$ (100\% of carbon) 
In the case of the positive response of types $\mathrm{BCR}_{2}$ (77\% glass, $23 \%$ carbon) and $\mathrm{BCR}_{3}(53 \%$ glass, $47 \%$ carbon) the plotted curves were divided into two parts, presented by two trend lines (1 and 2) for each curve. The reason for this division is the sharp slope of the curves in the interval from 0 to 0,1 for type $\mathrm{BCR}_{2}$ (77\% glass, $23 \%$ carbon) and 0 to 0,2 for type $\mathrm{BCR}_{3}(53 \%$ glass, $47 \%$ carbon) as a function of the strain ( $\varepsilon$. The corresponding trend equations established in this study are further shown in Table 4, as well as the resulting squared regression values and gage factors in Table 5.

The fractional resistance change was expressed from the linear trend equations as a function of the strain for each of the three types of the BCR rods, for the loading and unloading part of the cycle (1 and 2) for both types of responses.

The overall linear relationships of the two parameters obtained in the work showed a high satisfactory level of BCR sensing capability.

As can be seen in Table 5, the gage factor for type $\mathrm{BCR}_{2}(77 \%$ glass, $23 \%$ carbon) is almost five times higher than that for the type $\mathrm{BCR}_{3}\left(53 \%\right.$ glass, $47 \%$ carbon) and $\mathrm{BCR}_{4}(100 \%$ carbon). The gage factor of type $\mathrm{BCR}_{3}(53 \%$ glass, $47 \%$ carbon $)$ is slightly higher.

Table 5. Gage factors (GF) and squared regression values $\left(\mathrm{R}^{2}\right)$ of established trend equations.

\begin{tabular}{|c|c|c|c|c|c|c|}
\hline \multirow{2}{*}{ Type } & \multicolumn{2}{|c|}{ GF } & \multirow{2}{*}{$\mathrm{Xm}$} & \multicolumn{2}{|c|}{$\mathrm{R}^{2}$} & \multirow{2}{*}{ Response } \\
\hline & $1 *$ & $2 *$ & & 1 & 2 & \\
\hline \multirow{3}{*}{$\mathrm{BCR}_{2}$} & 0,58100 & 0,46312 & 0,52206 & 0,98965 & 0,99602 & \multirow{2}{*}{ Positive } \\
\hline & 0,09554 & 0,12951 & 0,11253 & 0,95651 & 0,97279 & \\
\hline & $-0,28869$ & $-0,34058$ & $-0,31464$ & 0,99218 & 0,97288 & Negative \\
\hline \multirow{3}{*}{$\mathrm{BCR}_{3}$} & 0,16095 & 0,15806 & 0,15951 & 0,98918 & 0,99668 & \multirow{3}{*}{ Positive } \\
\hline & 0,03847 & 0,07018 & 0,05433 & 0,98339 & 0,98172 & \\
\hline & $-0,14084$ & $-0,16380$ & $-0,15232$ & 0,93925 & 0,96892 & \\
\hline \multirow{2}{*}{$\mathrm{BCR}_{4}$} & 0,03581 & 0,03702 & 0,03415 & 0,97118 & 0,99305 & Positive \\
\hline & $-0,07451$ & $-0,07684$ & $-0,07568$ & 0,99448 & 0,98699 & Negative \\
\hline
\end{tabular}

The gage factor, known as the strain-sensing factor, shows the sensing behavior of the BCR samples. It increases with the decreasing of the carbon fiber percentage. This means that type $\mathrm{BCR}_{2}(77 \%$ glass, $23 \%$ carbon) has the most reliable monitoring behavior.

It is interesting to compare the resulted gage factors of the braided composite rods with other materials for the same applications. For example in a study of a carbon nanotube strain sensors, it was investigated that the range of the gage factor was between 1 and 5, depending on the percentage of the single walled carbon nanotube (SWCNT) polymer composites, and better sensitivity was established in the range of 3 to $10 \mathrm{wt} \%$ of the SWCNT in the polymer (Kang, 2006).

Another study, investigating a multi walled carbon nanotube (MWCNT) films used as strain sensing material, reported that the calculated gage factors were 2, 3,09 and 3,76, respectively, for 3 types of samples (Li, 2008).

Taking these examples into comparison with the investigated samples in this study, it is evident that the gage factors calculated for the BCRs are all less than 1, much smaller than the example studies set forth above. The reason for these small values of the gage factors would be, as mentioned, the higher percentage of the carbon component. On the other hand, the main advantage of the present materials is the superior reinforcing capabilities and therefore the combination of reinforcing and sensing capabilities.

\section{CONCLUSION}

This work represents a contribution to develop monitoring systems for civil engineering structures in order to improve their sustainability. The final goal of this study is to develop such online monitoring system, by the application of braided reinforced composite rods (BCR) in concrete structures.

This paper reported on the influence of carbon fiber amount in the sensing proformance of braided reinforced rods using glass and carbon. It was proven that all three types of BCR used can stand as a self-sensing material. The electrical contact set-up was effective in the purpose 
of resistance stabilization and measurent.Two types of responces were obtained by the BCR. Positive GF, in the case of the carbon fibreplaced inthe area subjected to tensile and negative GF, in the case of the carbon fibre placed in the compressive side of the rod. Furthermore, the GF increased with decreasing carbon fiber content. The most reliable monitoring behavior was given by type $\mathrm{BCR}_{2}(77 \%$ glass, $23 \%$ carbon) with the smallest carbon fiber content.

\section{REFERENCES}

Bakis, C. E., Nanni, A., Terosky, J. A., Koehler, S. W., 2001, Self -monitoring, pseudo - ductile, hybrid FRP reinforcement rods for concrete applications. Comp Sci and Tech 61, 815 - 823.

Gonilho Pereira, C., Fangueiro, R., Jalali, S., Araújo, M., Pina Marques, P. ,2008, Tensile performance of braided composite rods for concrete reinforcement; Challenges for Civil Construction, Porto.

Chung, D. D. L., 1997, Self-monitoring structural materials. Composite Materials Research Laboratory, State University of New York at Buffalo, Buffalo, NY 14260-4400, USA.

Kang, I., et al, 2006, Introduction to carbon nanotube and nanofiber smart materials. Composites: part B 37, March, 382-394.

Li, X., Levy, C., Elaadil, L., 2008, Multiwalled carbon nanotube film for strain sensing. Nanotechnology 19, $045501(7 \mathrm{pp})$.

Muto, N., Arai, Y., Shin, S. G., Matsubara, H., Yanagida, H., Sugita, M., Nakatsuji, T., 2001, Hybrid composites with self-diagnosing function for preventing fatal fracture. Comp Sci and Tech 61, 875883.

Nanni, F., Auricchio, F., Sarchi, F., Forte, G., Gusmano, G., 2006, Self - sensing CF-GFRP rods as mechanical reinforcement and sensors of concrete beams. Smart Mater Struct 15, 182-186.

Nanni, F., Ruscito, G., Forte, G., Gusmano, G, 2007, Design, manufacture and testing of self-sensing carbon fibre - glass fibre reinforced polymer rods. Smart Mater Struct 16, 2368-2374.

Okuhara, Y., Matsubara, H., 2005, Memorizing maximum strain in carbon - fibre reinforced plastic composites by measuring electrical resistance under pre - tensile stress. Comp Sci and Tech 65, 2148-2155.

Okuhara, Y., Matsubara, H., 2007, Carbon - matrix composites with continuous glass fibre and carbon black for maximum strain sensing. Carbon 45, 1152-1159. 